between January 2011 and January 2021, with a diagnosis of germ-line BRCA $1 / 2$ mutation and who underwent breast cancer risk-reducing surgery. The occurrence of occult carcinoma was analysed. A descriptive study of these patients was performed. All statistical analysis was performed with Stata/IC 13.0 for Windows.

Result(s)* During the study period a total of 168 patients with BRCA $1 / 2$ mutation were diagnosed and breast risk-reducing surgery was performed in 81 of them (48.2\%).

In $61.7 \%(n=50)$ of the cases, prophylactic surgery was performed after the diagnosis of bilateral breast cancer. Bilateral breast and in $7.4(n=6)$ ovarian cancer. In 58.0\% ( $n=47)$ the mutation was BRCA 1 and in $42 \%(n=34)$ BRCA 2. In $39.5 \%(\mathrm{n}=32)$ the mastectomy performed was nipple sparing and in $60.5 \%(n=49)$ simple. Breast reconstruction was performed after surgery in $93.8 \%(n=76)$ of cases. Definitive histopathological examination showed ductal carcinoma insitu in $3.7 \%(\mathrm{n}=3)$ of cases, and infiltrating carcinoma in $1.2 \%$ $(\mathrm{n}=1)$.

Conclusion* In our case series, approximately half of the patients carrying BRCA mutation have opted for breast cancer risk-reducing surgery, with a proportion of incidental carcinomas between $1-3 \%$ in the mastectomy surgical specimens. Therefore, we can conclude that in patients carrying BRCA1/2 mutation, prophylactic mastectomy is effective in reducing the risk of breast cancer.

\section{BREAST CANCER IN BRCA1 AND BRCA2 MUTATIONS CARRIERS, CLINICOPATHOLOGICAL FEATURES}

A Laura, E Felipe Pardo, J Montero Olmeda, MDLR Oliver*, A Sofia, SM Consuelo, M De Miguel Reyes. Hospital Universitario 12 de Octubre, Gynecologycal and Obstetrics department, Breast unit, Madrid, Spain

\subsection{6/ijgc-2021-ESG0.282}

Introduction/Background* Mutations in the BRCA1 or BRCA2 susceptibility genes are associated with most hereditary breast cancers with an identified pathogenic variant. These genes are implicated in about $15 \%$ of women with familial breast cancer. The aim of this study is to evaluate clinicopathological features of breast cancer diagnosed in BRCA1/2 mutations carriers.

Methodology A retrospective descriptive study of all women diagnosed with BRCA gene mutations at a high-volume center between January 2007 and October 2020 was performed. Patients' history of breast and ovarian cancer was collected. IBM SPSS Statistics ${ }^{\circledR}$ v25.0 was used for statistical analyses.

Result(s)* We included 165 patients diagnosed with BRCA1 and BRCA2 mutation, of which 91 women were diagnosed with breast cancer $(58.8 \%$ among BRCA1 carriers and $51.8 \%$ among BRCA2 carriers). The mean age at diagnosis was 41.4 (SD 9.6) and 42.7 (SD 10.5) years for BRCA1 and BRCA2 respectively $(p=0.54)$. In 32 women, risk-reducing mastectomy (RRM) was performed without previous diagnosis of breast cancer.

In $87 \%$, the histological type was infiltrating ductal carcinoma (IDC), and the most frequent intrinsic subtypes were, for BRCA1, pure-HER2 (70\%), luminal B (11\%) and triplenegative (6.8\%), and for BRCA 2, Luminal HER2 (39\%), Luminal B (25\%) and pure-HER2 (19.4\%).
No differences in stage at diagnosis were found between patients with BRCA1 and BRCA2 ( $\mathrm{p}=0.073$ ) with $39.4 \%$ and $36.7 \%$ of cases of lymph node involvement respectively $(p=$ 0.701).

Chemotherapy was used as primary treatment in 32\% of patients with an anatomopathologic complete pathologic response rate in $47 \%$ of cases. The response to chemotherapy was similar in both types of germline mutation $(p=0.722)$. Conclusion* To be a BRCA mutation carrier is associated with an increased risk of breast cancer at earlier ages than the general population, with no significant differences between the two types of mutation. The most frequent intrinsic subtype was different between the two groups while the stage at diagnosis was similar.

\section{CORRELATION OF BREAST CANCER TUMOR SIZE BY MAGNETIC RESONANCE IMAGING, ULTRASONOGRAPHY AND MAMMOGRAPHY ACCORDING TO MENOPAUSAL STATUS}

${ }^{1} \mathrm{C}$ Stanciu Pop*, ${ }^{2} \mathrm{~V}$ Veys, ${ }^{2} \mathrm{C}$ Van De Merckt, ${ }^{2} \mathrm{~S}$ Drisis, ${ }^{2} \mathrm{M}$ Radermecker, ${ }^{2} \mathrm{M}$ Moreau ${ }^{2}$ D Larsimont, ${ }^{2}$ CF Pop. ${ }^{1}$ CHU UCL Namur - Site Godinne, Pathology, Yvoir, Belgium; ${ }^{2}$ Institut Jules Bordet, Bruxelles, Belgium

\subsection{6/ijgc-2021-ESG0.283}

Introduction/Background* Breast cancer (BC) tumor size (TS) at diagnosis is one of the most important prognostic factors and represent a pivotal key driver of treatment planning for patients with BC. The aim of this study was to evaluate the place of complementary breast magnetic resonance imaging (MRI) for preoperative TS evaluation in patients with early stage BC according to menopausal status.

Methodology We performed a retrospective review of a prospectively collected database of patients treated by primary surgery at a single institution for unifocal invasive BC who had complete documentation of the tumor size from mammography (MMG), ultrasonography (US), and MRI. Pearson's correlation (rs) coefficients are reported with their linear relationships.

Result(s)* A total of 752 women with 757 BC tumors were analyzed. Median age was 61.1 years (range from 26 to 89). There were $591(78.6 \%)$ women with a postmenopausal status. Invasive ductal carcinoma (IDC) was present in $84 \%$ of tumors. The mean (SD) histopathological TS was 15.2 (8.3) $\mathrm{mm}$. The tumor size varied by imaging method: $13.7(7.3)$ mm for MMG, 13.1 (7.4) $\mathrm{mm}$ for US, and 16.4 (9.1) $\mathrm{mm}$ for MRI. Correlations between TS assessed by the breast imaging technique (BIT) and by histopathology were 0.80 for MRI, 0.75 for US, and 0.69 for MMG. There was an rs of 0.72 for MRI and US in premenopausal women while the rs for MMG was 0.64 , in the same group of patients. The correlations between the final pathology and BIT in postmenopausal patients were 0.83 for MRI, 0.76 for US, and 0.70 for MMG.

Conclusion* Correlations between tumor size determined by BIT and final pathological size remained higher with MRI than US or MMG examination. Menopausal status may influence the accuracy of TS estimation, with a better correlation in postmenopausal women for all BIT. 